\title{
Image Restoration and Enhancement Using Unified Blind Method
}

\author{
Seema Singh ${ }^{1}$, Vivek Singh ${ }^{2}$, Mohit Kapoor ${ }^{2}$, Raju Nandi ${ }^{2}$, Arup Dash $^{2}$ \\ ${ }^{1}$ Associate Professor, Dept. of Electronics \& Comm. Engg., BMS Institute of Technology, Bangalore, India. \\ ${ }^{2}$ Students, Dept. of Electronics \& Comm. Engg., BMS Institute of Technology, Bangalore, India.
}

\begin{abstract}
Images play a vital role in our day to day life therefore the quality and the information contained in an image is very important to be preserved for various process used in different applications such as military, medical science, astronomy etc. But while capturing an image, the degradation of an image such as blurring effect, noise, aliasing etc. takes place due to various environmental effects. This paper focuses on removing the degradation effects using the Unified Blind Method involving Multi Image Super Resolution and Multi/Single image blur de-convolution techniques. The approach makes use of alternating minimization (AM) procedure of a new cost function with respect to the unknown High Resolution (HR) image and Blur. The regularization term for the HR image is based upon the Huber Markov Random Field model (HMRF). The Blur estimation process makes use of the edge detection process, which improves the quality of blur estimate. For better estimation Fast Fourier Transform (FFT) is used to get the image in frequency domain. The regularization term for the blur is Gaussian (L2 Norm). The processing time of reconstruction using SR method is reduced by separating the interpolation process from registration process. The Unified Blind Method is tested on images and the efficiency of the method is verified.
\end{abstract}

Keywords: blur de-convolution; super resolution; blind estimate; Huber Markov Random field

\section{Introduction}

Many applications require clear undistorted image. They are medical imaging, security purposes and remote sensing. Traditional image processing methods are bulky and costly. In contrast, computational imaging systems use a more efficient and cost effective method for HR image generation. Degrading elements like blurring, aliasing and noise may affect the finest detail of an image. Spatial resolution, Blur de-convolution (BD) and super-resolution (SR) are the techniques being implemented to enhance the image. The difference being that blur deconvolution removes noise and blur, while super resolution removes the overlapping or aliasing effects from an image. Consequently, the input and output images in BD are of equal size, while size of the output image is increased in SR.

\section{A. Image Processing Basics}

An image may be defined as a two-dimensional function, $f(x, y)$, where $x$ and $y$ are spatial (plane) coordinates, and the magnitude of $f$ at any pair of co-ordinates $(x, y)$ is called the intensity or gray level of the image at that point. An image is called a digital image having $\mathrm{x}, \mathrm{y}$ and $\mathrm{f}$ value are discrete and finite. A digital image is comprised of a finite element number, each of which has a specific value and location. These elements are called picture elements or pixels or image elements. The three types of computerized processes are as follows:

- Low-level processing: Involves image preprocessing which reduces noise, contrast enhancement, and image sharpening.

- Mid-level processing: Involves image segmentation, description of objects and recognizing individual objects.

- High-level processing: Involves correct placing of an ensemble of recognized objects and image analysis.

B. Image Formation

Image $=$ PSF $*$ Object function + noise

- PSF: This describes the way information on the object function is spread as a result of recording the data. It is a characteristic of the instrument used to capture images (i.e. camera) and is a deterministic function (that operates in the presence of noise).

- Object function: This describes the object (or scene) that is being imaged and the way light is reflected from that structure to the imaging instrument 
- Noise: This is a nondeterministic function which can, at best, only be described in terms of some statistical noise distribution (e.g. Gaussian). Noise is a stochastic function which is a consequence of all the unwanted external disturbances that occur during the recording of the image data.

- Convolution operator * : A mathematical operation which 'smears' (i.e. convolves) one function with another.

Here, the function of the light reflected from the object/scene (object function) is transformed into the image data representation by convolution with the PSF. This function characterizes the image formation (or capture) process. The process is affected by noise.

The PSF is a characteristic of the imaging instrument (i.e. camera). It represents the response of the system to a point source in the object plane, as shown in Figure 2.1, where we can also consider an imaging system as an input distribution (scene light) to output distribution (image pixels) mapping function consisting both of the PSF itself and additive noise.

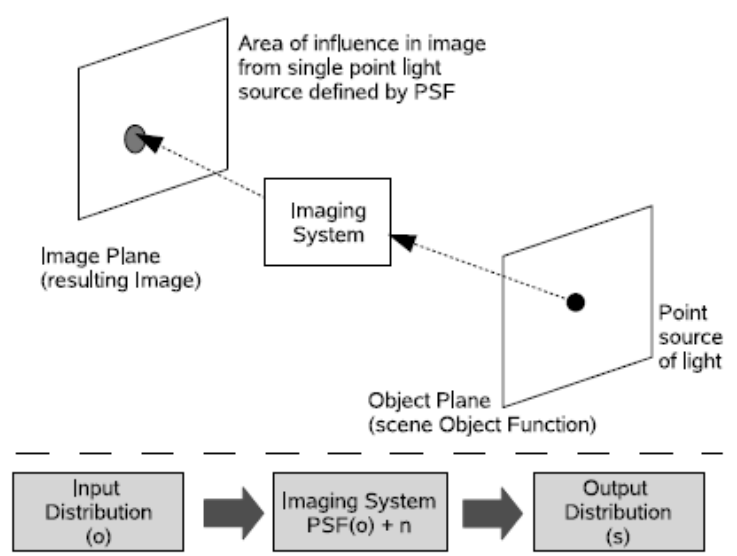

Fig. 1: An overview of the image formation 'system' and the effect of the PSF

\section{Types Of Images}

Images are classified based on the intensity levels in image, number of bits in an image, number of components in an image etc.

Binary images: A 2D array image in which each pixel has only two numerical values (0\&1). Typically the two colors used for a binary image are black and white: black corresponds to zero (background pixel) and white corresponds to one (foreground pixel).

Grayscale or intensity image: Grayscale images are often the result of measuring the intensity of light at each pixel in a single band of the electromagnetic spectrum. The intensity of a pixel ranges from 0 to 1 , with any fractional values in between . There are 0-255 levels of a gray scale.

Color images: A digital color image consists of three components i.e., red, green, and blue. So each pixel of image has three color channels corresponding to each red, green and blue color. There are 8x3=24 bits per pixel. Commonly used color space is RGB. Apart from this, there are HSV, YCbCr used in other context.

The color is expressed as an RGB triplet $(r, g, b)$, each component of which can vary from zero to a defined maximum value. If all the components are at zero, black is the result; if all are at maximum, the result is white. A secondary color is formed by the sum of two primary colors of equal intensity: cyan $=$ green + blue, magenta $=$ red + blue and yellow $=$ red + green $($ CMY color model $)$.

\section{Image Sampling}

It is important to understand how images can be sampled and how that relates to the various neighborhoods that can be used to process an image.

Rectangular sampling - In almost every case, images are sampled by laying a rectangular grid over an image as illustrated in Fig. 4.

Hexagonal sampling - An alternative sampling scheme is shown in Fig. 4 and is termed as hexagonal sampling. 


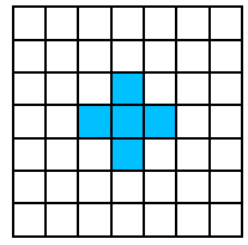

Rectangular sampling

4-connected

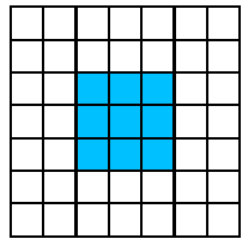

Rectangular sampling

8 -connected

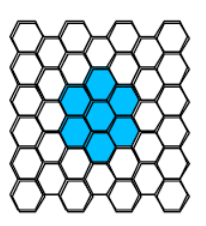

Hexagonal sampling 6-connected

Fig. 2: Digital image of sampling neighbour

\section{E. $\quad$ Noise in Images}

Image noise is random variation of brightness or color information in images, and is usually a part of electronic noise. It can be produced due to poor functioning of the circuitry \& sensor of a scanner or digital camera. Image noise can also arise in film grain $\&$ in the unpreventable shot noise of an ideal photon detector.

Gaussian noise: During acquisition, Gaussian noise is added to digital images. eg. Sensor noise arises due to poor illumination, transmission and high temperature. The noise is additive in nature, independent at every pixel $\&$ also independent of the signal intensity, caused mainly by thermal noise, including reset noise of capacitors. Amplifier noise is a primary portion of the "read noise" in an image sensor, that is, of the steady noise level in dark image areas.

Salt and Pepper Noise: "Impulsive" noise is called salt-and-pepper noise or spike noise. Salt-and-pepper noise image will have dark pixels in bright regions and bright pixels in dark regions. It can be eliminated by using interpolating around dark/bright pixels and dark frame subtraction.

Shot Noise: A statistical quantum fluctuation that basically is variation in the number of photons sensed at a given exposure level creates dominant noise in the lighter parts of an image from an image sensor. This noise is known as photon shot noise. Shot noise is different at different pixels.

\section{F. Resolution of an image}

Resolution quantifies how close lines can be to each other and still be visibly resolved. Resolution units can be related to physical sizes (e.g. lines per mm, lines per inch), to the overall size of a picture (lines per picture height), or to angular subtenant.

Pixel Resolution: Pixel is fundamental unit of digital image. Resolution is dependent on the size of pixel. Smaller is the pixel size, higher is the resolution and a clearer object in image is obtained.

Spatial Resolution: The column (C) by row (R) dimensions of the image defines the number of pixels used to cover the visual space captured by the image. This leads to the sampling of the image signal and is known as the digital resolution or pixel of the image. It is commonly written as C_R (e.g. 640_480, 800_600).

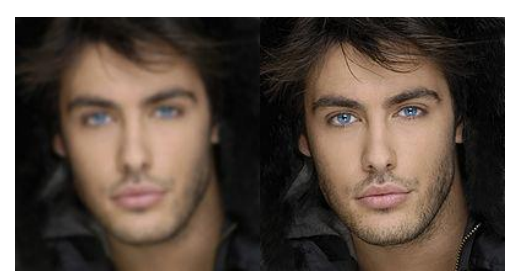

Fig.3: Image of different spatial resolution

Spectral Resolution: Color images distinguish different spectrum of light. Multispectral images distinguish finest differences of wavelength or spectrum than is required to reproduce color.

Temporal Resolution: High speed and movie cameras can identify events at different instances of time. The resolution of time in movies is normally 24-48 frames per second (fps), whereas high-speed cameras maybe equal to or more than 50 to $300 \mathrm{fps}$.

Radiometric Resolution: Radiometric resolution estimates how finely a system may represent or distinguish intensity differences and is normally written as a number of bits or a number level, for eg. 8 bits or 256 levels that is usually the case for computer image files. 
Blur: Blurring an image usually makes the image out of focus. Blurring, in signal processing, is usually obtained by convolving the image with a low pass filter. In Fig. 7, the amount of blurring is increased by increasing the pixel radius.

\section{Existing Methods}

Multi-image super-resolution (MISR) forms an HR image by fusing many LR images [1]-[5]. While, single-image super-resolution (SISR) methods, also known as learning-based, patch-based or example-based SR techniques[6]-[8], replace small spatial patches in a LR image by taking extracts from many HR images. SISR methods do not need motion and blur estimation processes in comparison to MISR methods, but have a lower performance. In case of Blur De-convolution (BD), the most proposed methods for reconstruction are from a single image (SIBD) [9]-[12]. However, multi-image BD (MIBD) methods [13]-[16] are also developed to boost the reconstruction performance. LR images given to a SR (MISR) system mostly have sub-pixel displacements between their fields of view (FOV). Also both SR and MIBD systems may either use LR images that have differences in their point spread functions (PSFs) due to variations in the parameters of the lens (such as aperture, focal length, and focus), or use LR images with variances in their illumination conditions (photometric variations) due to dissimilar camera parameters (such as exposure time and aperture size). Most of the works on $\mathrm{BD} / \mathrm{SR}$ are non-blind, i.e. they do not explicitly consider blur identification during the reconstruction procedure. Many studies assume that the PSFs are fully known a priori due to capturing images under controlled environmental/imaging conditions. Other works assume that the amount of blur is negligible and can be omitted from the reconstruction [7]. While these simplifications are valid under certain circumstances, they are impractical for many real-world applications in which varying blurring effects may accompany their imaging process. In parallel to these works, others have studied blur identification along with $\mathrm{BD} / \mathrm{SR}$. These papers are classified into two categories: methods that consider blur identification and image restoration as two disjointed processes [9], [11], [18], [19] and methods that combine these two processes into a unified procedure, e.g. alternating minimization (AM).

\section{Unified Blind Procedure}

In unified blind method both the BD and SR techniques are being used to enhance the resolution of image(s).

Unified procedure [20] for single and multi-image super resolution, Single and multi image blind Deconvolution. Regularizing cost function leads to estimation of HR image and blurs. High resolution image is obtained by applying unified blind procedure on colour/gray scale images. Performance measurent parameters are MSE and PSNR. BSR algorithm is used to regularize the energy function with respect to the original image and blur. It is implemented in both the image \& blur domains. Adaptive BSR algorithm is the one in which the cost function is based on Alternating Minimization approach, the regularization term for image is huber markov random field and for blur is Gaussian (L2 norm).

a. Edge Detection: Edge detection is the name for a set of mathematical methods which aim at identifying points in a digital image at which the image brightness changes steeply or, in other words, has discontinuities. The regions where image brightness changes sharply are typically organized into a set of curved line segments known as edges. The same problem of locating discontinuities in 1D signal is known as step detection and the problem of finding signal discontinuities over time is known as change detection.

b. Blur Estimation: The blurred image is modeled as a convolution between the original image and an unknown point-spread function. Motion blur occurs when there is relative motion between the camera and the object being captured, blur that occurs when the motion has constant speed and a fixed direction.

The aim is to find out the angle \& length of the blur. Once the angle \& length of the blur are identified, a point spread function can be formed. This point spread function is then used in direct de-convolution methods to help restore the degraded image. Blur estimation in the filter domain is more efficient than the pixel domain. The first fact is supported by preprocessing the reconstructed image using an edge emphasizing smoothing operation which aims to enhance soft edges toward step edge while smoothing out weak structures.The second fact is achieved by setting large values for the regularization coefficients of both the HR image and the smoothing function in the initial iterations, and decreasing these values gradually at every iteration. The last fact suggests the use of the gradients of the LR and preprocessed HR images instead of their pixel values for the blur estimation. By the use of Gaussian (L2-norm) prior(s) for the blur(s), the blur estimation procedure will be solely based on convolution and multiplication-by-constant operations, and so the blur(s) can be updated fast by pixel-wise multiplications and divisions in the frequency domain. 
Alternating minimization procedure is used in which either the image or blur is fixed and is minimize the other variable with respect to the first variable. The Huber markov random field is used as regularization term in blind de-convolution. It removes distorting artifacts from the image.

Interpolation based method is the form of super resolution technique used to increase the clarity of the image. Lanczos3 resampling or Lanczos filter is a mathematical formula used to interpolate the value of a digital signal between its samples. It maps each sample of the given signal to a translated and scaled copy of the Lanczos kernel, which is a sinc function windowed by the central hump of a dilated sinc function. The sum of these translated and scaled kernels is then evaluated at the desired points.

\section{Proposed Method}

First part of the work is on synthetic image. The image to be enhanced is resized to $256 \mathrm{x} 256$ pixels. A user defined PSF is convoluted with the image to get a blurred image. The size of the PSF matrix is $21 \mathrm{x} 21$. Fast fourier transform is used to reduce the processing time as it uses its symmetric property to use only half of the matrix. Fast fourier transform is also used for blur estimation procedure will be solely based on convolution and multiplication-by-constant operations, and so the blur(s) can be updated fast by pixel-wise multiplications and divisions in the frequency domain. The Gaussian L2 norm is the regularization term for blur estimation. This blurred image is then convoluted with an initialized matrix to get an estimate of the blur to be removed from the distorted image. The values in the initialized matrix can be between $0 \& 1$. This step is iterated 14 times. The matrix is updated at the end of each iteration. The MSE is calculated by comparing each image with previous iteration. The estimated PSF (at the end of the $10^{\text {th }}$ iteration) is de-convoluted (blind de-convolution) with the blurred image and the parameters. Blind de-convolution removes the blur and noise from the distorted image to form a de-blurred image. This de-blurred image is enhanced by removing the aliasing effects and increasing the size of the image (Super resolution). In case of videos, each frame of a video is treated as an image. Each frame is stored after the completion of 14 iterations. These frames are then run simultaneously to provide a distortion free video.
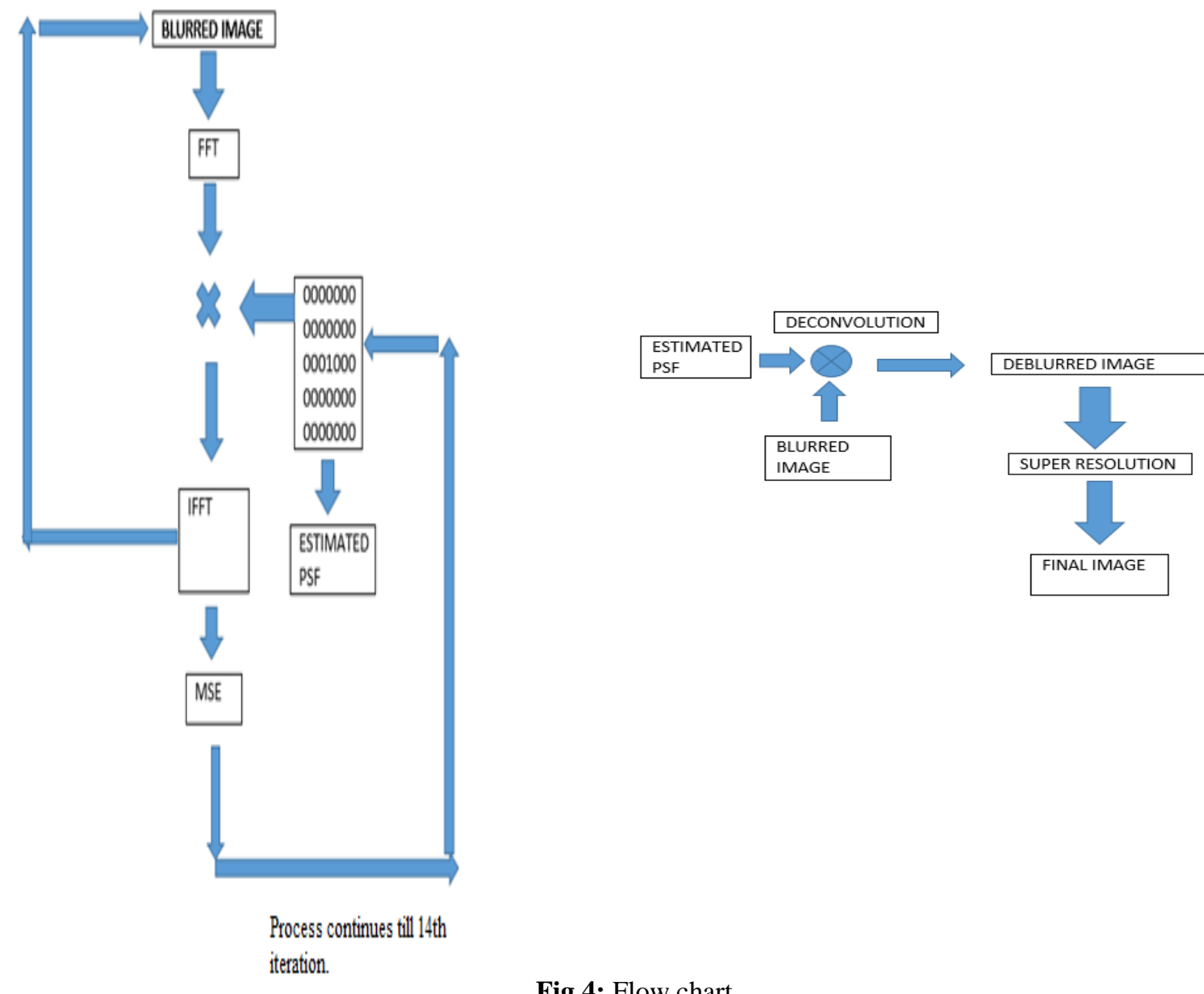

Fig.4: Flow chart 


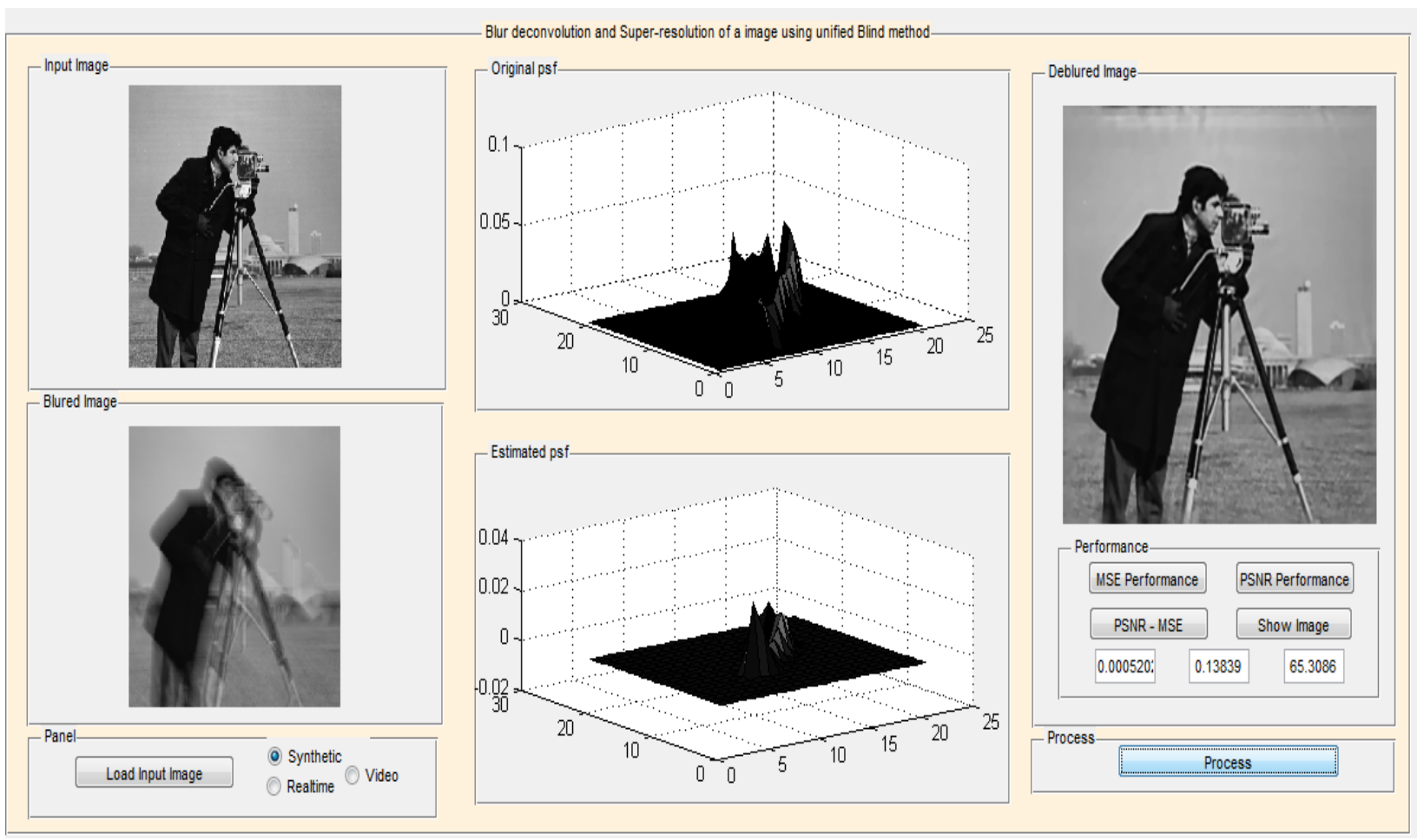

Fig.5: GUI showing the Original PSF and the Estimated PSF along with the MSE and PSNR values.

\section{Discussion On Results}

The above shown figure represents the graphical user interface of the result window. There are 3 radio buttons representing synthetic and real time image and video options. In this example a synthetic image is used. The load input image is a push button where the user needs to browse and select an image of their choice. The input image is grayscale 256x256 image. The original PSF shows the graph for the values in the $21 \times 21$ matrix. These are random user defined values.

The blurred image is shown in Fig. 5. It is created by convoluting original image with the PSF. The estimated PSF graph represents the mathematical value of the amount of PSF (blur) present in the blurred image.

This estimated PSF is used to de-blur the image by clicking the process push button. The blurred image undergoes the blind convolution and super resolution techniques. In the Deblurred image column we get the final estimate of the original image. Performance is indicated by MSE and PSNR. Following four attributes are used to analyze the results.

- $\quad$ MSE performance gives the graph of mean square error after each iteration

- $\quad$ PSNR performance gives the peak signal to noise ratio in dbs after each iteration

- $\quad$ PSNR-MSE give the comparison of mean square error vs peak signal to noise ratio.

- $\quad$ The show image button compares the Blurred image and Deblurred image in a separate window.

In addition to above, there are three text boxes in the generated GUI which shows the following:

- $\quad$ The first text box represents the mean value of the estimated PSF. In this case, it is 0.0052 .

- The second text box represents the Mean Square Error in the de-blurred image. In this case, it is 0.0073 .

- $\quad$ The third text box represents the PSNR value of the de-blurred image. In this case, it is $65.3086 \mathrm{db}$.

If number of iterations is increased after 14, the image gets distorted because further improvement cannot be achieved. Plot of Fig. 6 shows the mean square error of the image against the number of iterations. It can be seen that the MSE of the image decreases with every iteration. The MSE at the end of the $14^{\text {th }}$ iteration is 0.0073 . 


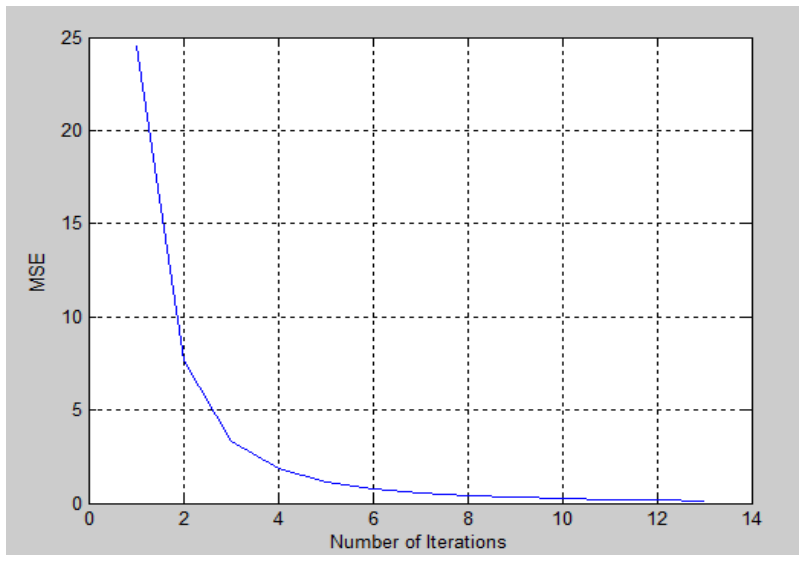

Fig. 6: MSE after each iteration

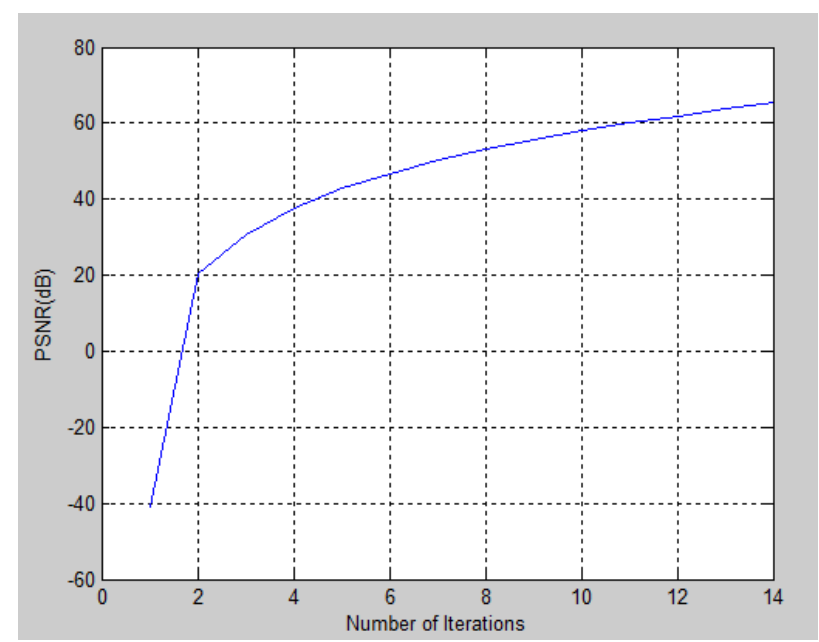

Fig. 7: PSNR after each iteration

The plot of Fig. 7 shows the PSNR of the image against the number of iterations. The PSNR increases with each iteration. The PSNR at the end of $14^{\text {th }}$ iteration is $65.3086 \mathrm{db}$. Fig. 8 shows plot indicating the MSE vs PSNR value for each value. As it can be seen in the figure, MSE and PSNR are inversely proportional to each other. The final values of MSE and PSNR are 0.0073 and $65.3086 \mathrm{db}$ respectively.

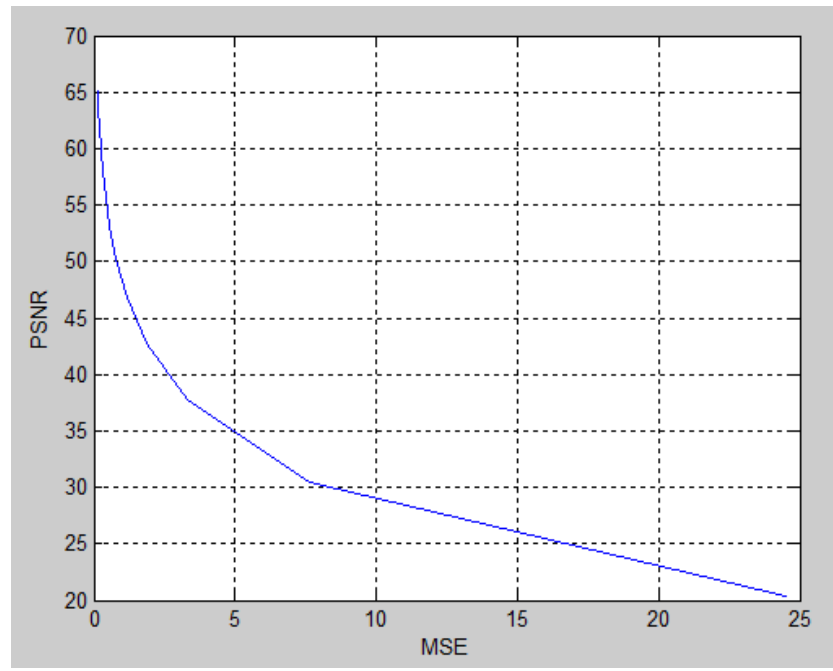

Fig 8: MSE vs PSNR after each iteration. 


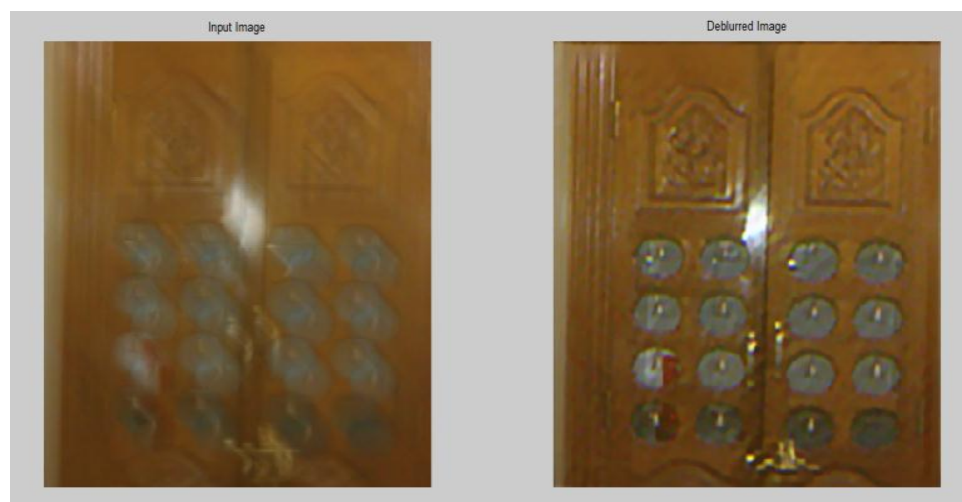

Fig. 9: Real time blurred input image and De-blurred image

Fig. 9 shows a case where a real time colored image is used to test the proposed method. The MSE value is 0.0062 and the PSNR value is $78.3 \mathrm{db}$ in this case.

A lot of difference can be seen between input blurred image and the De-blurred image. Idol's image which is not visible in left is clearly visible on the right side of the figure. This clearly shows the efficiency of the proposed method. It enhances the image and optimizes it visually.

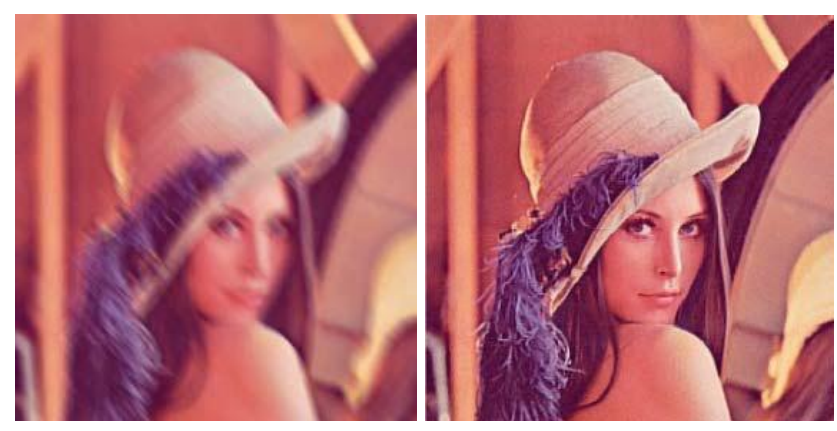

Fig.10: Unified blind algorithm applied on Lena image

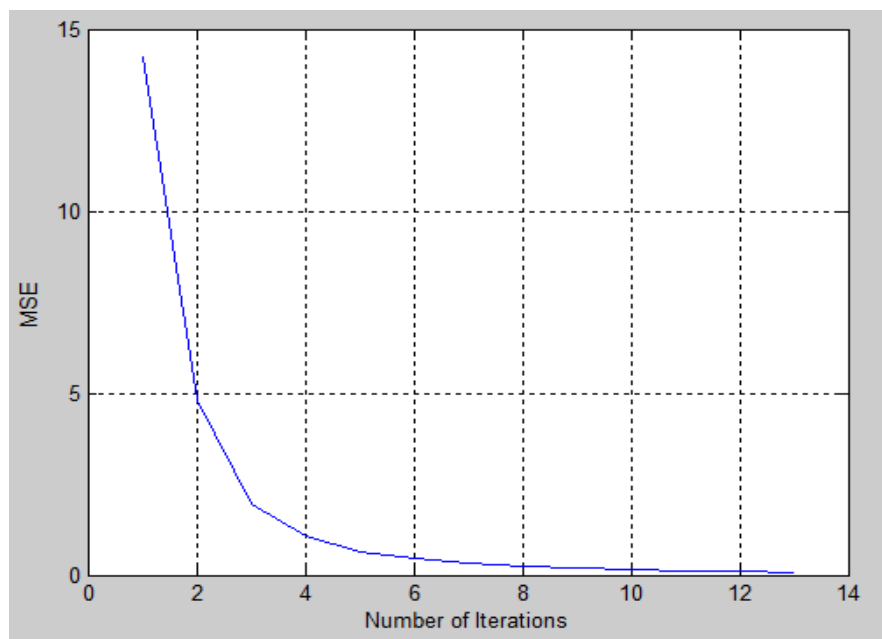

Fig.11: MSE after each iteration. 


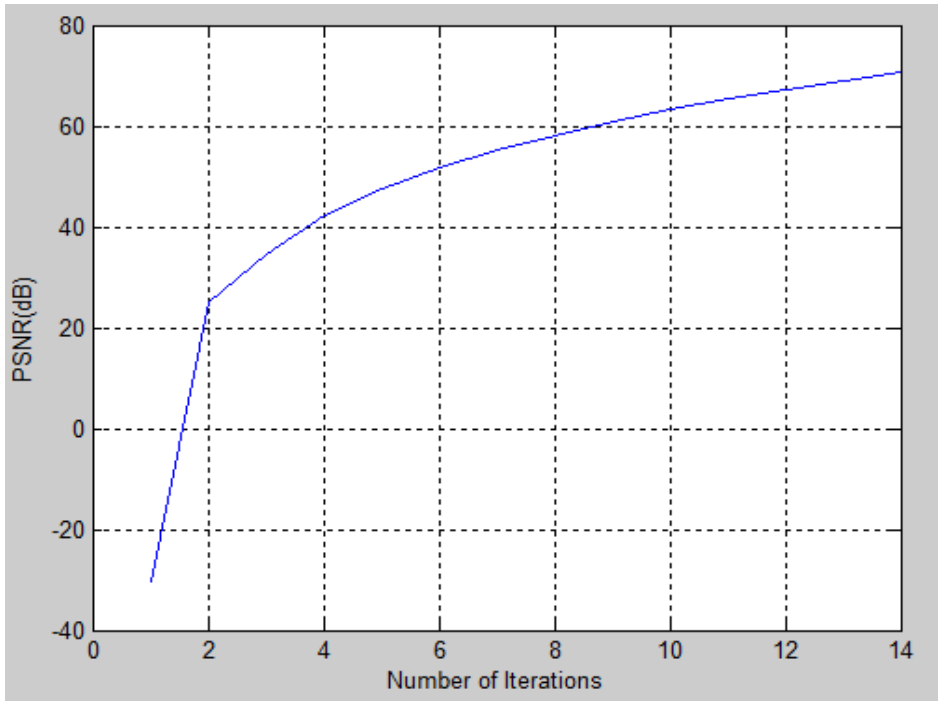

Fig.12: PSNR after each iteration.

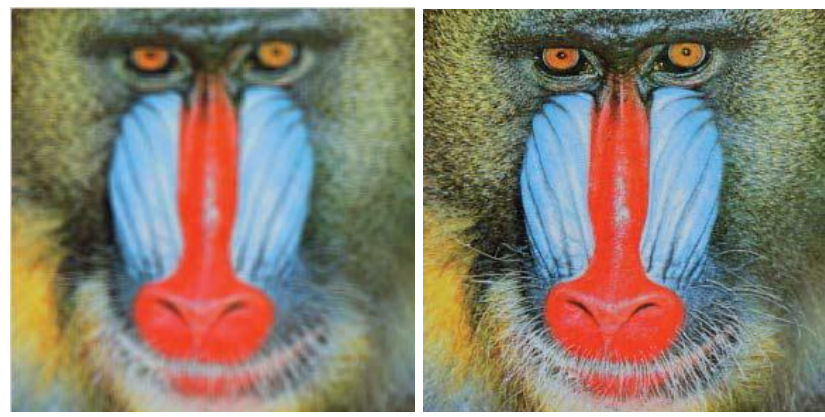

Fig.13: Unified blind algorithm applied on mandrill image

Fig. 10 shows a synthetic coloured image. The MSE value is 0.0344 and the PSNR value $70.67 \mathrm{db}$. Fig. 13 shows a case of synthetic coloured image. The MSE value is 0.0043 and the PSNR is $68.879 \mathrm{db}$. The MSE and PSNR values have been improved in comparison with the existing results. After 14 iterations the image gets distorted resulting in high MSE and low PSNR values.

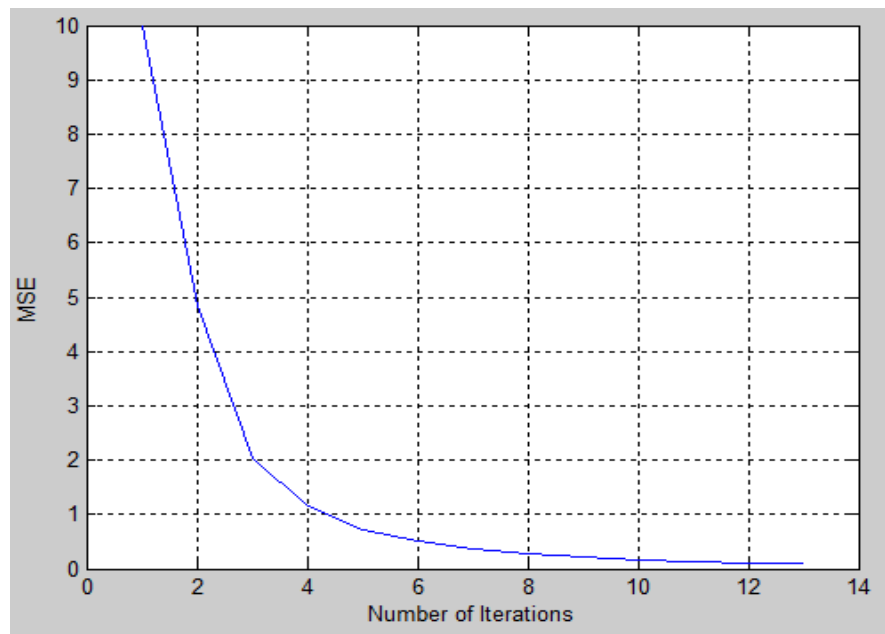

Fig.14: MSE after each iteration. 


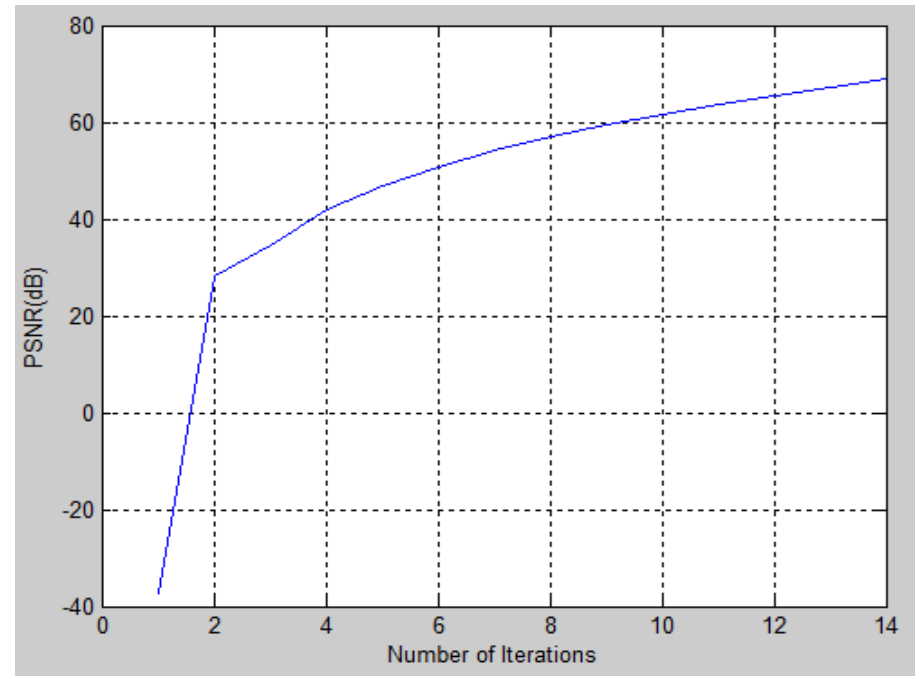

Fig.15: PSNR after each iteration.

A high pass filter in addition to the previous technique is used to filter out noise more substantially. The parameters values (gamma correction, alpha corrections, beta corrections) have been enhanced. These enhancements result in a clearer and more optimized output image.

Table 1: Comparison for MSE and PSNR

\begin{tabular}{|c|c|c|c|c|}
\hline S.No & Cases & Parameters & Esmaeil et al [20] & Proposed Method \\
\hline \multirow{2}{*}{1} & \multirow{2}{*}{ Fig. 5 } & MSE & 0.008 & 0.0073 \\
\cline { 3 - 5 } & & PSNR (db) & 29.5 & 65.3086 \\
\hline \multirow{2}{*}{2} & \multirow{2}{*}{ Fig. 10 } & MSE & 0.04 & 0.0344 \\
\cline { 3 - 5 } & & PSNR (db) & 32.5 & 70.67 \\
\hline \multirow{2}{*}{3} & \multirow{2}{*}{ Fig. 13 } & MSE & 0.0045 & 0.0043 \\
\cline { 3 - 5 } & & PSNR (db) & 28.3 & 68.879 \\
\hline
\end{tabular}

However the processing time is increased, which is the main limitation. The same method has been applied on videos. However the processing time for videos is considerably high as all the frames for videos have to undergo processing. For a $30 \mathrm{fps}$ video, there are 30 images (frames) to be processed in comparison to one frame in an image. Hence for multiple seconds videos, the processing time is very high.

\section{Conclusion}

A blind unified method for multi-image super-resolution (MISR), single-image blur deconvolution (SIBD), and multi- image blur deconvolution (MIBD) is presented. The optimization procedure is based on alternating minimization of a well- defined cost function which consists of a L2 fidelity term, a HMRF prior for the HR image, and a L2 prior for each of the blur functions. By contrast, our MISR (or SR) method accepts a number of LR images with sub pixel displacements but the same blur function and noise parameters. The assumption of blur and noise similarity in SR allows us to separate the registration and upsampling processes from the reconstruction procedure. The proposed blur estimation procedure preprocesses the estimated HR image by applying an edge emphasizing smoothing operation which enhances the soft edges toward step edges while smoothing out weak structures of the image. The parameters are altered so that more and more salient edges are contributed in the blur reconstruction at every iteration. For better performance, the blur estimation is performed in the filter domain using the derivatives of the preprocessed HR image and the LR image(s).

This work can be extended in several directions, for instance to have space-variant blur identification, study joint image registration and restoration procedures, or consider compression errors in the forward model. De-blurring of the video on the same has been implemented but work can be extended to reduce the processing time. 


\section{References}

[1]. M. Irani and S. Peleg, "Improving resolution by image registration," Graph. Models Image Process., vol. 53, pp. 231-239, Apr. 1991.

[2]. R. C. Hardie, K. J. Barnard, J. G. Bognar, E. E. Armstrong, and E. A. Watson, "High resolution image reconstruction from a sequence of rotated and translated frames and its application to an infrared imaging system,” Opt. Eng., vol. 37, no. 1, pp. 247-260, 1998.

[3]. R. C. Hardie, K. J. Barnard, and E. E. Armstrong, "Joint map registration and high-resolution image estimation using a sequence of undersampled images,” IEEE Trans. Image Process., vol. 6, no. 12, pp. 1621-1633, Dec. 1997.

[4]. S. Farsiu, M. Robinson, M. Elad, and P. Milanfar, "Fast and robust multiframe super resolution," IEEE Trans. Image Process., vol. 13, no. 10, pp. 1327-1344, Oct. 2004.

[5]. R. Schultz and R. Stevenson, "Extraction of high-resolution frames from video sequences," IEEE Trans. Image Process., vol. 5, no. 6, pp. 996-1011, Jun. 1996.

[6]. W. Freeman, T. Jones, and E. Pasztor, “Example-based super-resolution,” IEEE Comput. Graph. Appl., vol. 22, no. 2, pp. 56-65, Mar.-Apr. 2002.

[7]. D. Glasner, S. Bagon, and M. Irani, "Super-resolution from a single image," in Proc. IEEE 12th Int. Conf. Comput. Vis., Sep. 2009, pp. 349-356.

[8]. W. Dong, L. Zhang, G. Shi, and X. Wu, "Image deblurring and superresolution by adaptive sparse domain selection and adaptive regularization,” IEEE Trans. Image Process., vol. 20, no. 7, pp. 1838-1857, Jul. 2011.

[9]. R. Fergus, B. Singh, A. Hertzmann, S. T. Roweis, and W. T. Freeman, "Removing camera shake from a single photograph," ACM Trans. Graph., vol. 25, pp. 787-794, Jul. 2006.

[10]. Q. Shan, J. Jia, and A. Agarwala, "High-quality motion deblurring from a single image," ACM Trans. Graph. (SIGGRAPH), vol. 27, no. 3, pp. 73:1-73:10, Aug. 2008.

[11]. S. Cho and S. Lee, "Fast motion deblurring," ACM Trans. Graph. (SIGGRAPH ASIA), vol. 28, no. 5, p. 145, 2009.

[12]. L. Xu and J. Jia, "Two-phase kernel estimation for robust motion deblurring," in Proc. 11th Eur. Conf. Comput. Vis. I, Ser., 2010, pp. $157-170$.

[13]. F. Šroubek and P. Milanfar, "Robust multichannel blind deconvolution via fast alternating minimization," IEEE Trans. Image Process., vol. 21, no. 4, pp. 1687-1700, Apr. 2012.

[14]. G. Harikumar and Y. Bresler, "Perfect blind restoration of images blurred by multiple filters: Theory and efficient algorithms," IEEE Trans. Image Process., vol. 8, no. 2, pp. 202-219, Feb. 1999.

[15]. F. Sroubek and J. Flusser, "Multichannel blind deconvolution of spatially misaligned images," IEEE Trans. Image Process., vol. 14, no. 7, pp. 874-883, Jul. 2005.

[16]. F. Sroubek and J. Flusser, "Multichannel blind iterative image restoration,” IEEE Trans. Image Process., vol. 12, no. 9, pp. 10941106, Sep. 2003.

[17]. E. Faramarzi, V. R. Bhakta, D. Rajan, and M. P. Christensen, "Super resolution results in PANOPTES, an adaptive multi-aperture folded architecture," in Proc. 17th IEEE Int. Conf. Image Process., Sep. 2010, pp. 2833-2836.

[18]. M. Chang, A. Tekalp, and A. Erdem, "Blur identification using the bispectrum," IEEE Trans. Signal Process., vol. 39, no. 10, pp. 2323-2325, Oct. 1991.

[19]. N. Nguyen, P. Milanfar, and G. Golub, "Efficient generalized crossvalidation with applications to parametric image restoration and resolution enhancement," IEEE Trans. Image Process, vol. 10, no. 9, pp. 1299-1308, Sep. 2001.

[20]. Esmaeil Faramarzi, Dinesh Rajan and Marc P. Christensen "Unified Blind Method for Multi-Image Super-Resolution and Single/Multi-Image Blur Deconvolution” IEEE Transaction On Image Processing, Vol. 22, No. 6, June 2013. 